\section{CYCLIC AMP}

\section{Kith and Kinuse}

from our Molecular Biology Correspondent

FOR those persons who regret the passing of vitalism, the nearest thing to the élan vital is nowadays cyclic AMP. It has been implicated in the control of an extraordinary variety of biological processes-most recently in visual excitation, where it is reported to encompass $a^{\prime}$ change in membrane permeability, following capture of a photon in a retinal rod (Bitensky, Gorman and Miller, Proc. US Nat. Acad. Sci., 68, 561; 1971).

The search for a cyclic AMP receptor protein in a number of systems has now turned up what may well prove to be a universally relevant mechanism. Gill and Garren (ibid., 786), for example, have isolated such a receptor from adrenal cortex cells, and find that it is associated with protein phosphokinase activity. This latter has for some time been known to be activated by the cyclic nucleotide. When the crude product is chromatographed on an ionexchange column, the two activities superimpose in the first 'peak, but thereafter barely separated peaks of acceptor and kinase activity are eluted. The first component also migrates electrophoretically as a single zone; but when cyclic AMP is added, it dissociates to give a fully active kinase that migrates independently, and is no longer affected by the AMP. This effect is substantially reversible: when the dissociated kinase and receptor are mixed in the absence of the nucleotide, they associate, with suppression of kinase activity. From an estimate of the molecular weights of the complex and its constituents a 1:1 stoichiometry is inferred. But the last peak eluted from the column, which is associated with high kinase and low acceptor activity (the residuum of the latter being eluted separately), seems to generate kinase dimers on dissociation.

In the same issue of the Proceedings, Erlichman, Hirsch and Rosen (ibid., 731) describe the isolation of a cyclic AMR-activated kinase from beef heart. In this case gel filtration or ion exchange chromatography gave a major and a minor peak of activity. When cyclic AMP was present the kinase activity peak was displaced to a position corresponding to lower molecular weight, and when recovered was no longer subject to control by the nucleotide. Again the dissociation was reversible: in the absence of cyclic AMP, the kinase and the residual fraction, which possessed binding activity, recombined, and the kinase once more became AMP-sensitive. A precisely similar situation was uncovered by Krebs's group (Reimann et al., Biochem.
Biophys. Res. Commun., 42, 187; 1971), working with rabbit skeletal muscle. Thus it is supposed that the system has the function of allowing the cyclic AMP concentration to control the kinase activity, which in turn governs the activity of a variety of enzymes. This mechanism was foreseen by Tao, Salas and Lipmann in an article published last year, and offers a new instance of an association between catalytic and regulatory subunits, of which the archetypal example is perhaps Gerhart's aspartate transcarbamylase.

The part played by phosphorylation with kinase in glycogen metabolism in muscle is the subject of the latest instalments in the great epic of glycogenolysis that is being unfolded by Krebs and his associates. Walsh et al. (J. Biol. Chem., 246, 1968; 1971) have shown that, in a word, the protein kinase phosphorylates phosphorylase kinase, which phosphorylates phosphorylase $b$ to give phosphorylase $a$. Any readers who have lost the thread of the narrative may wish to be reminded that phosphorylase kinase, which operates on muscle phosphorylase, is strongly activated on being itself phosphorylated. This process is promoted by cyclic AMP, but a second and slower activation mechanism exists, which is independent of cyclic AMP, and is shown to involve autophosphorylation of the phosphorylase kinase (an enzyme that requires ATP, but not cyclic AMP). Walsh et al. (ibid., 1977) have also discovered at specific protein inhibitor that associates with the cyclic AMP-dependent kinase, but does not bind the nucleotide. Isolation and enzymatic characterization of the kinase are described by Reimann $e t$ al. (ibid., 1986). Two components with similar activity are present, as in the kinases from other tissues. They offer the suggestion, based on the low substrate specificity, that the same enzyme may be responsible for phosphorylating all the many proteins in different tissues, the activity of which is controlled by this means.

\title{
New Lower Limit for Quasar Masses
}

IT now seems firmly established that the mass of a quasi-stellar object must exceed $10^{7}$ solar masses. In the next issue of Nature Physical Science, D. P. Whitmire and C. N. Davids describe yet another method of estimating this important parameter. Their method is based on the observation that in many QSOs with both emission and absorption lines, the absorption redshifts are greater than the emission redshifts, presumably because the absorption lines arise in material previously ejected from, but now falling back upon, the QSO. The ejected material evidently failed to reach escape velocity, so the relative velocity of the shell and QSO, which is given by the difference in the absorption and emission redshifts, permits an estimate of the minimum mass interior to the shell, provided that the shell radius is known. Their estimate of $2 \times 10^{7} M_{\odot}$ for the mass of the quasar PHL 5200 is of particular interest because they have calculated the minimum shell radius directly from a change in the absorption and emission redshifts which seems to have taken place over a period of one year; the estimated mass limit is found to be independent of the source distance.

No assumption is made about the cosmological nature of QSOs, and only the difference in the emission and absorption redshifts is regarded as necessarily a genuine velocity shift. Their limit of $2 \times 10^{7} M_{\circ}$ agrees with earlier estimates based on the assumption that QSOs lie at cosmological distances and is in particularly good agree- ment with those estimates based on general energy considerations which give lower limits of $10^{8}$ and $10^{7} \mathrm{M}_{\odot}$, depending on whether the energy source is nuclear or gravitational.

The lower limit to a QSO mass of $\sim 10^{7} M_{\odot}$ based on optical observations will be very much an underestimate for the total mass associated with a QSO if, for instance, the optical activity represents activity in the nucleus of a galaxy. The similarity of the structure of radio sources associated with QSOs and with radio galaxies suggests that this may indeed be the case and it would therefore be of interest to have a reliable upper mass limit. The methods which have been proposed and which seem capable of yielding a useful upper limit are all based on finding a QSO in a cluster of galaxies and comparing its redshift with that of the associated cluster members. Finding genuine QSOs in clusters of galaxies does not, however, seem to be the easiest of occupations, and an estimate of $M \leq 5 \times 10^{13} M_{\odot}$ by Bachall and Salpeter (Astrophys. J. Lett., 158, L15 ; 1970), based on the object B264, was criticized by Barnothy (Astrophys. J. Lett., 159, L133; 1970) and is now known to be irrelevant because B264 has now been shown to be not a QSO but a compact galaxy. Nevertheless, at least some QSOs may occur in clusters and this in itself would seem to imply a limit of $10^{13}$ to $10^{14} M_{\odot}$, for it seems unlikely that the mass of a cluster member exceeds the mass of a typical cluster. 